\title{
Key Technical Study of Infrared Thermography on Aerodynamic Heating Measurement in Hypersonic Wind Tunnel
}

LI Ming, ZHU Zhi-wei,LI ZHI-hui

( China Aerodynamics Research \& Development Center, Mian-yang city ,621000, China)

\begin{abstract}
In the article, it was discussed how to correct surface directional emittance in a big optical incident angle and how to relate model coordinates to corresponding pixel locations of model infrared mapping and how to raise the accuracy of heat transfer rates measurement. Some simple practical methods were obtained. As the example of these methods to be applied , the transfer rates on a thin skin flat plate with a wedge and on sphere-cylinder model were measured by means of infrared thermography or thermocouple. The infrared results and thermocouple ones agreed with each other very well.
\end{abstract}

\section{Introduction}

Aerodynamic heating experimental techniques have been one of important research contents to hypersonic aerodynamics. To develop an aircraft, heat protection design of an airship, returned satellites and other hypersonic vehicles is very important. If contact method is used to measure heat flux on complicated shape aircraft, it is difficult to fix heat flux sensor and to obtain satisfied result. In the meantime, the existence of these sensors easily breaks shape and surface roughness of the model and disturbs flow field. infrared thermography has the characteristic of non-contact measurement, not disturbing flow field and intuitionistic and visual image. By means of it, thousands of data on model surface can be obtained at a test.

Throughout over 40 years, infrared thermography has been one of main methods of aerodynamic heating measurement in wind tunnel experiment. As aerodynamic test diagnosis tool, infrared thermography technique already has been used extensively for measuring instantaneous temperature, catching eddy, judging boundary layer transition and separation flow etc.. Gartenberg, Roberts A.S had looked back application of infrared thermography in the wind tunnel test about 25 years and thought that infrared thermography technique had become a kind of normal measurement means ${ }^{[1]}$.

Y.Le Sant summarized the application of infrared thermography at large wind tunnels at ONERA and particularly introduced the application in high enthalpy wind tunnel ${ }^{[2]}$.Bynum from American Engineering Development Center(AEDC) achieved heat transfer coefficient on the cone and hemisphere cylinder model at the VKF wind tunnel B through infrared thermography ${ }^{[3]}$. At the same time, he still analyzed the change in heat transfer coefficient and boundary layer transition on the cone model under the condition of having or not boundary layer mixing lines, and compared these results with theory one.

Kamran Daryabeigi successfully made use of infrared thermography technique to measure surface temperature and aerodynamic heating on hemisphere cylinder model at Langley-31 Wind Tunnel in the United States in the temperature range of $20 \sim 200{ }^{\circ} \mathrm{C}$, and analyzed the test accuracy ${ }^{[4]}$. Deng Jian-ping from China used infrared thermography technique at the electric arc wind tunnel, and expanded the temperature range to $3500{ }^{\circ} \mathrm{C}{ }^{[5]}$.

For all that, some key techniques of aerodynamic heating experiment using infrared thermography in hypersonic wind tunnel not are fully solved, such these problems as temperature measurement under great measurement angle, the accuracy to be improved, the corresponding relation between model spatial coordinates and the ones in infrared thermography etc.. In the text, the study of these problems is carried on and some practical and simple methods are acquired.

\section{Temperature measurement under great measurement angle}

The model surface emittance is the function of the wave-length, temperature, measurement angle and surface condition 
etc.. In some measurement angle, the surface emittance is constant. While exceeding certain measurement angle, the surface emittance sharply diminishes. Therefore, the model surface emittance must be corrected.

Variational relation was given between surface emittance of insulate material and measurement angle In literature [4]:

$$
\varepsilon(\theta)=\frac{2\left(\mathrm{n}^{2}-\sin ^{2} \theta\right)^{0.5} \cos \theta\left\{1+n^{2} /\left[\cos ^{2} \theta\left(\left(n^{2}-\sin ^{2} \theta\right)^{0.5}+\sin \theta \operatorname{tg} \theta\right)^{2}\right]\right\}}{\left[\left(n^{2}-\sin ^{2} \theta\right)^{0.5}+\cos \theta\right]^{2}}
$$

If knowing the refractive index $n$ of model material, one can calculate surface emittance under different measurement angle $\theta$. But one hardly knows the refractive index $n$ of the opaque material.

Measures have been taken to insure model surface interested regions inside the sound measurement angle. A few of infrared windows are fixed in the wind tunnel wall in Russia so that surface temperature of model interested region can be measured against with infrared camera, shown as figure 1. But this method demands changing the angle of infrared window at any time according to the pose of model, which is very inconvenient.

In the text, a device, which is put inside the test section of wind tunnel, and made infrared camera sealed and can go round and round, is adopted. Through the device, measurement angle between ray axes of infrared camera and the normal of model surface interested regions is adjusted as possibly as small so that the emittance of measured surface is constant, shown as figure 2. Because of no effect blocked by wind tunnel wall, the device can be quickly moved to adjust measurement angle. This method is economy, simple, safe, practical.

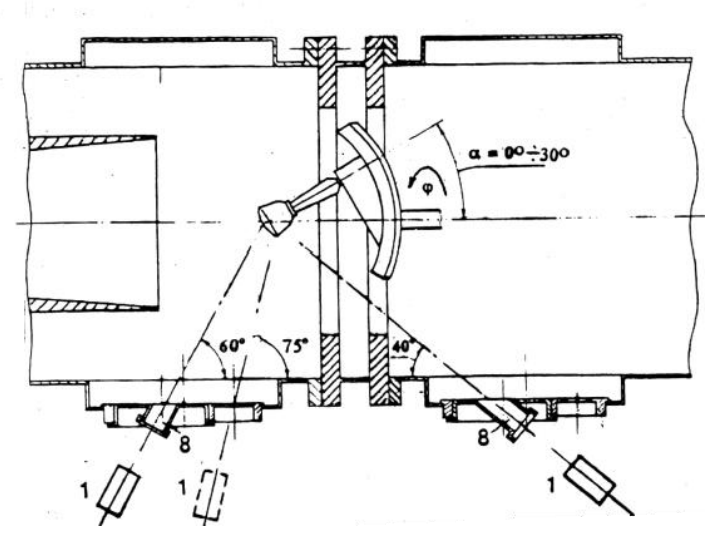

Fig.1 Measurement sketch of a few infrared windows

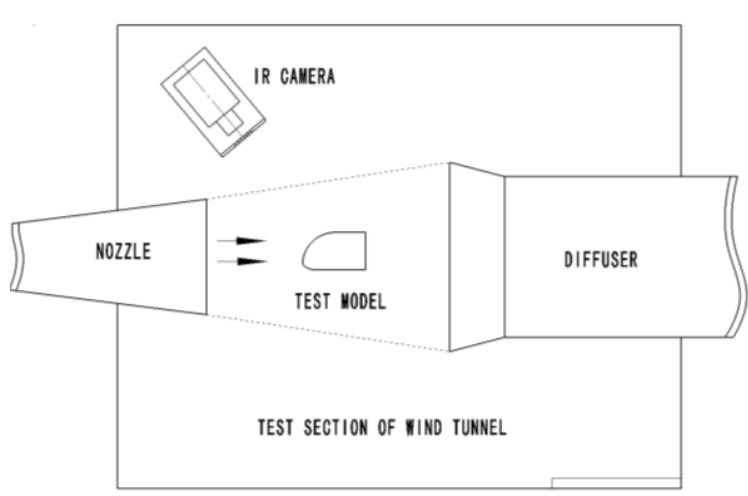

Fig. 2 Rotated and sealed device diagram

For all that, because some model head is located at flow field center, surface temperature of model head can not be accurately measured using infrared camera even if the above-mentioned method is adopted. For this, an reflecting infrared ray system is applied to measure surface temperature of model head, shown as figure 3. By means of changing measurement angle of infrared window and infrared reflector so that the measurement angle is less than $60^{\circ}$. So accurate temperature measurement of model head can be insured.

\section{Other way to raise accuracy of heat transfer rates measurement}

When knowing model surface emittance, one can deduce the model surface temperature $T_{w}$ from infrared radiation received by infrared camera. To nonmetal model material, the data approach is a thick wall method. Through solving one dimensional instantaneous heat conduction equation, one can get: 
In the equation (2),

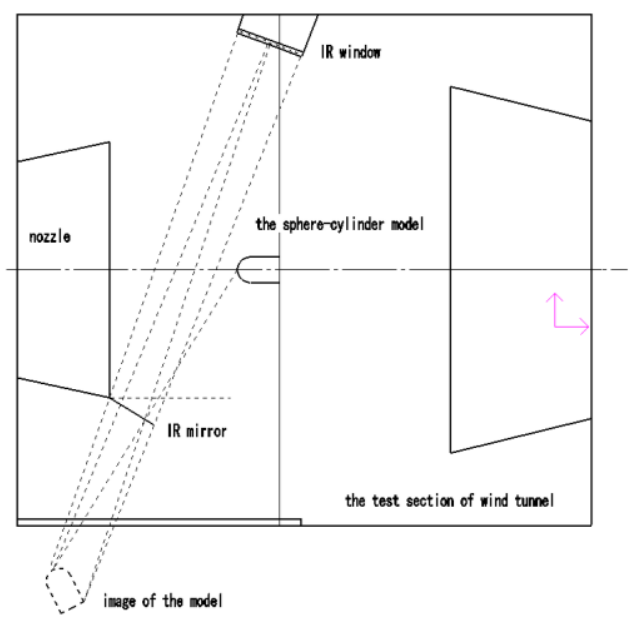

Fig. 3 reflecting infrared ray system diagram

$$
\frac{T_{w}-T_{i}}{T_{a w}-T_{i}}=1-\exp \left(\beta^{2}\right) \operatorname{erfc} \beta
$$

$$
\beta=h(t / \rho c k)^{0.5}
$$

When knowing each item at the left side of equation (2), then one can work out the value $\beta$, thus can get surface heat transfer coefficient $h$ and surface heat flux Q :

$$
Q=h\left(T_{a w}-T_{w}\right)
$$

In the equation (2)- (4), the $T_{w}$ is a model surface temperature, $\mathrm{K} ; T_{i}$ is a model initial temperature, $\mathrm{K} ; T_{a w}$ is an adiabatic temperature, $\mathrm{K} ; t$ is a model heating time, $\mathrm{s} ; \quad h$ is heat transfer coefficient, $\mathrm{W} / \mathrm{m}^{2} . \mathrm{K} ; \rho c k$ is density, specific heat and heat conduct coefficient of the model material respectively.

In the equation (2), through solving the equation through heat transfer coefficient $h$ against these five parameters $T_{w} 、 T_{a w}$ $T_{i},\left(\rho C_{p} k\right)^{1 / 2}, t$, and using the Taylor series method, one can get the random uncertain degree of the heat transfer coefficient [6].

$$
\frac{\Delta h}{h}= \pm \frac{1}{h}\left\{\sum_{i=1}^{5}\left[\left(\frac{\partial h}{\partial P_{i}}\right)^{2} \cdot\left(\Delta P_{i}\right)^{2}\right]^{2}\right\}^{\frac{1}{2}}
$$

Among them, $\Delta h$ is the uncertain degree of $h ; \Delta P_{i}$ is the uncertain degree of $P_{i}$. After obtaining partial derivative of these five parameters and putting them into equation (5), one can obtain the final uncertain degree of the heat transfer coefficient:

$$
\frac{\Delta h}{h}= \pm\left\{\begin{array}{l}
\left\{\left[\left(\frac{Q T_{w}}{2\left(T_{a w}-T_{w}\right)}\right) \cdot\left(\frac{\Delta T_{w}}{T_{w}}\right)\right]^{2}+\left[\left(\frac{Q(1-Z) T_{a w}}{2\left(T_{a w}-T_{w}\right)}\right) \cdot\left(\frac{\Delta T_{a w}}{T_{a w}}\right)\right]^{2}\right]^{\frac{1}{2}} \\
+\left[\left(\frac{Q Z T_{i}}{2\left(T_{a w}-T_{w}\right)}\right) \cdot\left(\frac{\Delta T_{i}}{T_{i}}\right)\right]^{2}+\left[\frac{\Delta(\rho c k)^{1 / 2}}{(\rho c k)^{1 / 2}}\right]^{2}+\left[0.5 \cdot \frac{\Delta t}{t}\right]^{2}
\end{array}\right\}
$$

In the equation (6): 


$$
\begin{gathered}
Q=\frac{\sqrt{\pi} Z}{\beta(\beta \sqrt{\pi} Z-1)} \\
Z=\frac{T_{a w}-T_{w}}{T_{a w}-T_{i}}=e^{\beta^{2}} \cdot \operatorname{erfc}(\beta)
\end{gathered}
$$

Seen from the equation (6), under the certain test condition, the random uncertain degree of the heat transfer coefficient is the function of model surface temperature $T_{w}$. The relation between the random uncertain degree of the heat transfer coefficient on lifting body model and model surface temperature is shown as figure 4 under the test condition of Mach 17.44 , total temperature $961.5 \mathrm{~K}$, total pressure $1.577 \mathrm{MPa}$ and attack of angle $30^{\circ}[7]$. The random uncertain degree of the heat transfer coefficient varies with the model surface temperature. Beyond the temperature range of $40 \sim 200{ }^{\circ} \mathrm{C}$, the heat transfer coefficient has a bigger error. But for a given experimental condition, an adiabatic temperature and heating time is certain, the model surface temperature is mainly decided by model material. Therefore one may choose reasonable model material, and control model surface temperature and raise the measurement accuracy of heat transfer rates.

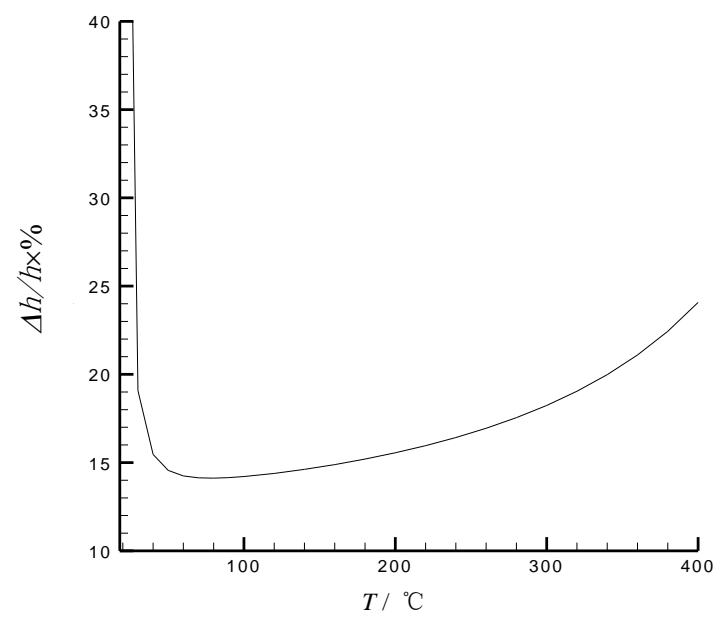

Fig.4 Relation of random uncertain degree of the heat transfer coefficient with surface temperature

\section{Corresponding relation between model spatial coordinates and the ones of infrared thermography}

In the past, it was limited that model spatial coordinates was deduced from the pixels coordinates of its infrared thermography according to the proportion of the model to its infrared thermography. In addition, model 3D reconstruction through infrared thermography is very difficult. In the article, a reference mesh method is used. That is, some reference points(lines) at the known position of the model surface are marked. From these reference points(lines) combined with image boundary probe, the spatial coordinates of a model can be quickly made certain from its corresponding infrared thermography. However, such these factors as the vibration, friction of quick injecting model equipment could have certain influence on the model spatial coordinates to be confirmed with a reference mesh method. For this reason, further improvement of the method is carried on by some scholars. For example, Y.Le Sant, through the reference points of a model surface, 3 parallel displace variables and 3 rotational variables of infrared camera relative to a model ,has made sure the actual spatial coordinates of a model from its corresponding infrared thermography ${ }^{[8]}$.

\section{Application of infrared thermography to aerodynamic heating measurement in hypersonic wind tunnel}

\subsection{Experimental equipment and instruments}


In the article, all experiments are carried out in a hypersonic low density wind tunnel. The hypersonic low density wind tunnel, a continuous equipment crossing flow region of imitating height $30 \sim 90 \mathrm{~km}$, is composed of a big power heating device, a steady room, nozzle, test section, diffuser, vacuum system etc. Its working medium is nitrogen and Mach number M5 M10, M12, M16 and M24, total temperature 300 1700 K, Renault number $\operatorname{Re}_{\infty} 4.4 \times 10^{4} / \mathrm{m} \sim 2.8 \times 10^{7} / \mathrm{m}$. The diameter of all nozzle exit is $300 \mathrm{~mm}$.

Before test, the model is placed to out of flow field. After stabilizing flow field, the model is quickly injected into flow field by quick model injecting equipment. The injecting time is about 0.25 second.

Experimental infrared window glass material is germanium, transmission wave band 8 12 $\mu \mathrm{m}$, which is opaque in visible light wave range. Through analyzing with Ansys software, maximal strain is located at the center of the germanium glass, which is about $5.31 \mu \mathrm{m}$, and corresponding strain is $0.634 \mathrm{Mpa}$, which is safety to the germanium glass.

The infrared camera thermovision 900 is made by Swedish AGEMA Company, its main function indexes is as follows:

- $\quad$ detector and working wave band: tellurium cadmium mercury, 8 12 mms;

- temperature measurement range:-40 ${ }^{\circ} \mathrm{C}-1500^{\circ} \mathrm{C}$;

- temperature measurement accuracy: $\pm 1 \%$;

- frequency:15 - 30/second;

- dynamic range:12 bits;

- pixel: $272 \times 136$.

\subsection{Results comparison of infrared thermography measurement with thermocouple one}

The instantaneous temperature on a thin skin flat plate with a wedge was measured by means of both infrared thermography and thermocouple in the hypersonic low density wind tunnel at the same time. At nominal test conditions of Mach 12, stagnation temperature 650K, stagnation pressure $1630 \mathrm{kpa}$, heat transfer rates on the thin skin flat plate model with a wedge were obtained. The infrared data agreed with the thermocouple data very well ${ }^{[9]}$. The sketch map of the thin skin flat plate model with a wedge was shown as Figure 5(a), heat transfer rates measured with the two methods were shown as figure 5(b) . In the Figure 5(b), IR denoted results obtained with infrared thermography, TC denoted ones with thermocouple, the A, B and C, D and E denoted measuring points at model surface, 0, 5, 15, 30 denoted angle of attack.
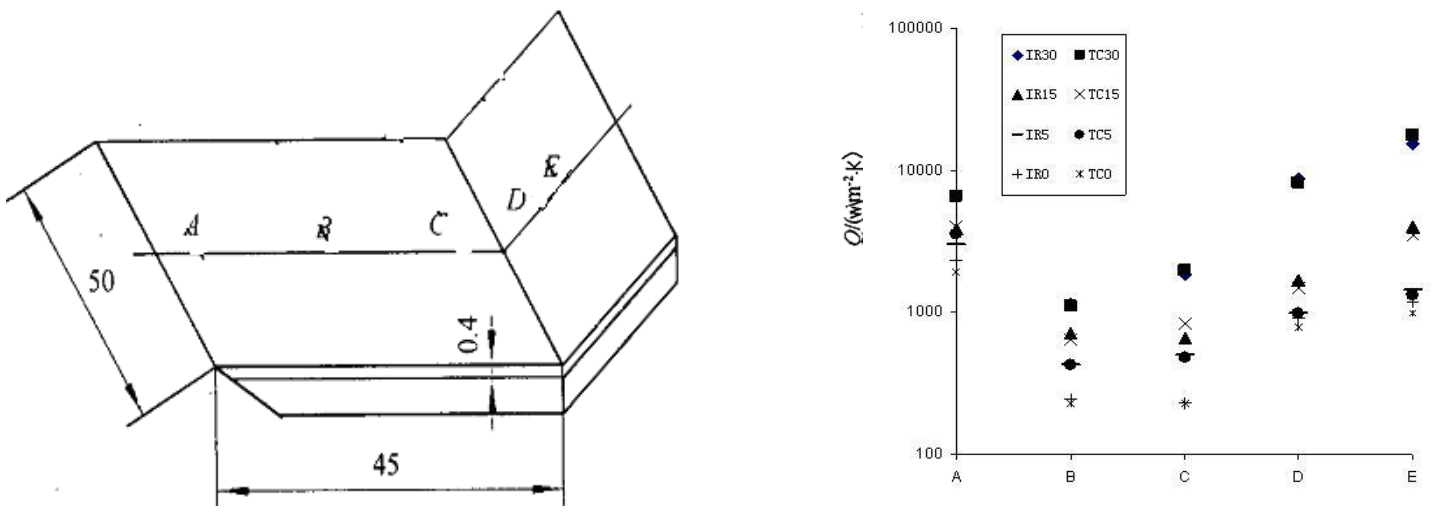

Fig. 5 (a) Sketch map of the model

Fig. 5 (b) Heat transfer rate comparison between two measuring methods

\subsection{Aerodynamic heating measurement on the hemisphere cylinder model}

Aerodynamic heating on sphere-cylinder model was experimentally studied using infrared thermograpy too. This was a classic model because aerodynamic heating distributions on the model were largely studied whether through experiment or 
through engineering calculation in the domestic and international. However, ONLY in the range of 30 90 of the model heat transfer rate distributions were given at most experiments. Heat transfer rate distributions in the range of $0^{\circ} \sim 90^{\circ}$ of the model were acquired by means of reflecting infrared ray system as fig.2. The infrared results were compared with DSMC computational ones and the results agreed with each other very well, shown as figure 6. Experimental conditions were as follows: Mach number: M16, attack angle: $0^{\circ}$, stagnation temperature: $943 \mathrm{~K}$, stagnation pressure: $2.9 \times 10^{6} \mathrm{~Pa}$. Among S/R, $\mathrm{S}$ denoted arc length of sphere-cylinder model, $\mathrm{R}$ denoted arc radius of the one.

\section{6 conclusion}

(1) Through rotated and sealed device of infrared camera and reflecting infrared ray system, the accurate temperature measurement can be obtained under great measurement angle condition.

(2) It can raise the measurement accuracy of aerodynamic heating to choose suitable model material according to a testing condition.

(3) By means of a reference mesh, the spatial coordinates of a model can be quickly made certain from its corresponding infrared thermography.

(4) Because surface emittance of metal model is lower, surface emittance of metal model can be increased through nigrescence. However, surface nigrescence causes easily the serious reflection. In addition, spraying black paint on a model can raise surface emittance too, but black paint shells off easily when surface temperature of a model is high. When the surface temperature is lower, measurement error of infrared thermography is bigger. These problems need further study.

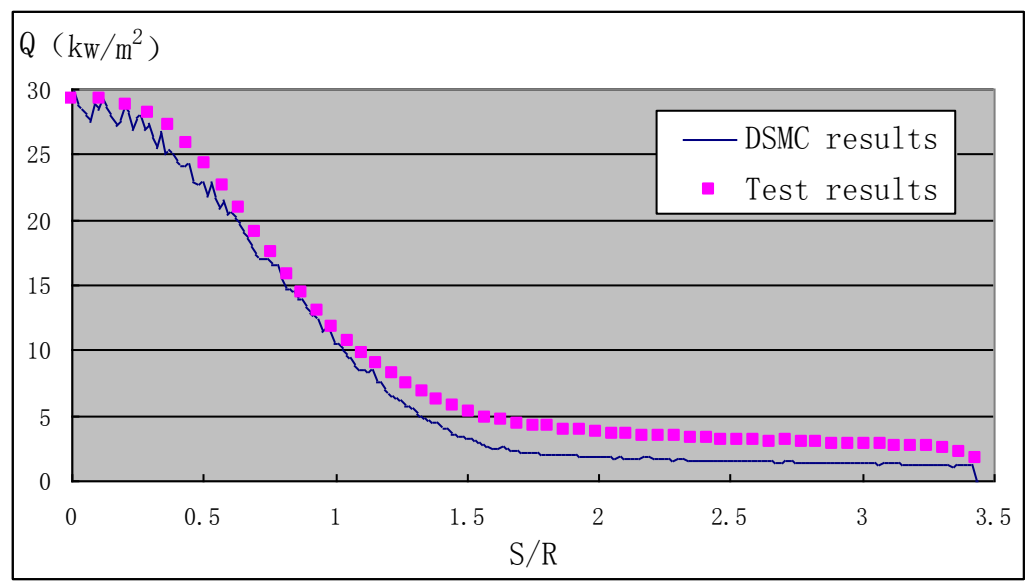

Fig. 6 Heat transfer rate comparison between test and DSMC computation

\section{REFERENCES}

[1] The E of Gartenberg, Roberts the A S. Twenty-five Years of Aerodynamics Research with Imaging.N91-20452, 1991

[2] The Y. Le Sant, M.Marchand, P. Millan, J. Fontaine.An Overview of infrared thermography Techniques Used in Large Wind Tunnels.ELSEVIER:Aerospace Science and Technology 6(2002)355-366.

[3] Bynum D S, Hube F K, Key C M, etc.Measurements and Mapping of Aerodynamic heating in VKF Tunnel B with an infrared Camera.AEDC-TR-76-54

[4] Kamran Daryabeigi.Global Surface Temperature|Heat Transfer Measurements Using infrared Imaging.AIAA 92-3959. 
[5] DENG Jianping, WANG Guolin, HUANG Peiran.The IR thermography technique for high temperature measurement.Experiments and Measurements in Fluid Mechanics[R], Vol.15No.1, Mar.,2001.

[6] BOYLAN D E,CARVER D B,STALLINGS D W,TRIMMER L L. Measurement and mapping of areodynamic heating using a remoting infrared scanning camera in continuous flow wind tun2 nel[R].AIAA 79-2799, 1978.

[7] ZENG Xue-jun,LI Ming,LIU Tai-kui,Li Si-xin2. The character research of aerodynamic heating on lifting body model using infrared thermographic technique. ACTA AERODYNAMICA SINICA[R], Vol.22,No.4, Dec.,2004.

[8] Y. Le Sant, B.Ddleglise, Y.Mebarki.An automatic image alignment method applied to pressure sensitive paint measurements.ONERA, 1997,10.

[9] LI Ming.Preliminary research of infrared thermographic technique in hypersonic low density wind tunnel.Experiments and Measurements in Fluid Mechanics[R], Vol.17,No.4, Sep.,2003. 